\title{
Application of Extracorporeal Membrane Oxygenation in Patients with Scrub Typhus Complicated with Acute Respiratory Distress Syndrome
}

\author{
Xiaozu Liao, ${ }^{1,2}$ Zhou Cheng, ${ }^{1,2}$ Liqiang Wang, ${ }^{1,2}$ Ziqiang Xu, ${ }^{1,2}$ Qiuyue Zheng, ${ }^{1,2}$ Rui Chen, ${ }^{3}$ Guisen $\mathrm{Wu},{ }^{3}$ \\ Binfei $\mathrm{Li}^{1,2}$ \\ ${ }^{1}$ Department of Extracorporeal Membrane Oxygenation Laboratory, Zhongshan City People's Hospital, Zhongshan, China; \\ ${ }^{2}$ Department of Anesthesiology, Zhongshan City People's Hospital, Zhongshan, China; ${ }^{3}$ Department of Emergency Intensive Care \\ Unit, Zhongshan City People's Hospital, Zhongshan, China
}

\section{ABSTRACT}

A 68-year-old woman was diagnosed with scrub typhus and acute pneumonia. Acute respiratory distress syndrome (ARDS) occurred on day 4 after admission and was treated with extracorporeal membrane oxygenation (ECMO). After 7 days of ECMO assistance, her respiratory condition gradually improved, and ECMO was removed. On day 20 after admission, she was discharged without any sequelae. The results suggest that ECMO should be considered as early as possible for patients with ARDS caused by scrub typhus.

\section{INTRODUCTION}

Scrub typhus is an acute fatal infection caused by tsutsugamushi rickettsia infection in the body. Tsutsugamushi rickettsia is an obligate intracellular bacterium (Orientia tsutsugamushi) with unique biological properties. Most cases occur in the tsutsugamushi triangle, which covers most of northern and eastern Asia, islands in the western Pacific, and parts of northern Australia [Kelly 2009]. Human infections are caused by bites of infected chigger mites. After the incubation period (6 to 21 days), there may be fever, headache, and discomfort [Kundavaram 2013]. Scrub typhus is characterized as a nonspecific febrile illness, or the patient may have body symptoms (fever, rash, myalgia, and headache) or dysfunction of organs such as kidney (acute kidney injury), lung (pneumonia), heart (myocarditis), liver (hepatitis), central nervous system (meningitis), or blood (thrombocytopenia syndrome) [Jayaprakash 2019; Premaratna 2013; Ki 2018; Vajpayee 2017; Adhikari 2018; Ra 2019]. Although most patients have rapid improvement after appropriate antibiotic treatment, a small number of patients have serious complications.

Pulmonary infections are common in patients with scrub typhus. The patients usually develop mild interstitial pneumonia that is self-limiting with timely treatment [Song 2004]. In severe cases, however, pulmonary pathology includes

Received December 6, 2019; accepted February 4, 2020.

Correspondence: Xiaozu Liao; Binfei Li, East Sunwen Road, Zhongshan City, Guangdong Province, 528403 China; (86)13450921219 (e-mail: liaoxiaozu@163.com;286025919@qq.com). pulmonary hemorrhage, pulmonary edema, vascular damage, and diffuse cellular infiltration, leading to acute respiratory distress syndrome (ARDS) [Jeong 2007]. ARDS is a lifethreatening disease.

Standard treatments include the application of a protective ventilation setting and prone position ventilation. If the patient is still hypoxic under this regimen, extracorporeal membrane oxygenation (ECMO) is an option [Fichtner 2018]. This article reports a case of scrub typhus complicated by ARDS, in which ECMO was a bridge to recovery.

\section{CASE REPORT}

A 68-year-old female patient was admitted to hospital because of weakness and muscle soreness for 1 week. The patient had always been healthy and had no obvious cause of the fatigue, muscle aches, and fever, but she had walked to the woods 1 week prior. The patient was seen in a nearby hospital, but the effect was not good, so she came to our hospital.

Admission examination showed body temperature $36.8^{\circ} \mathrm{C}$, heart rate 98 beats/min, respiratory rate 26 breaths $/ \mathrm{min}$, blood pressure $95 / 53 \mathrm{mmHg}$, and blood oxygen saturation 96\%. Ambiguous findings were Glasgow Coma Scale 12 points; Acute Physiology and Chronic Health Evaluation II 16 points; and dilated pupils, diameter $3 \mathrm{~mm}$ and sensitive to light, with mild yellow staining of the sclera. A $1.5 \times$ $1.5-\mathrm{cm}$ eschar was visible on the right shoulder (Figure 1). Chest $\mathrm{x}$-ray examination showed lung exudation (Figure 2).

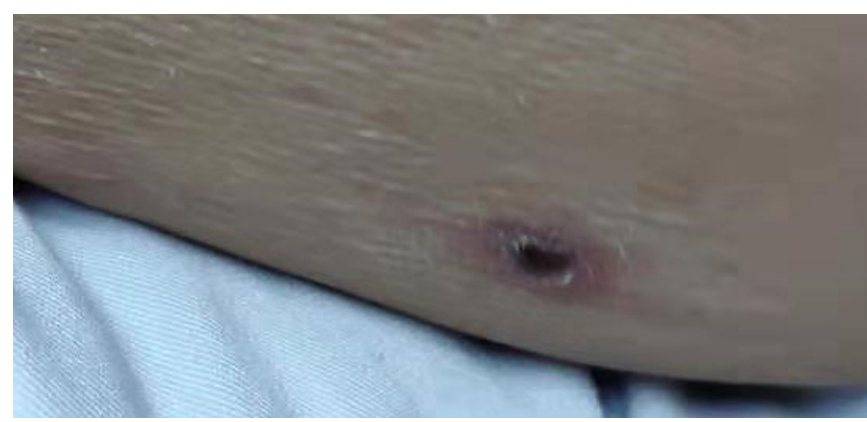

Figure 1. Patient's right shoulder eschar. 


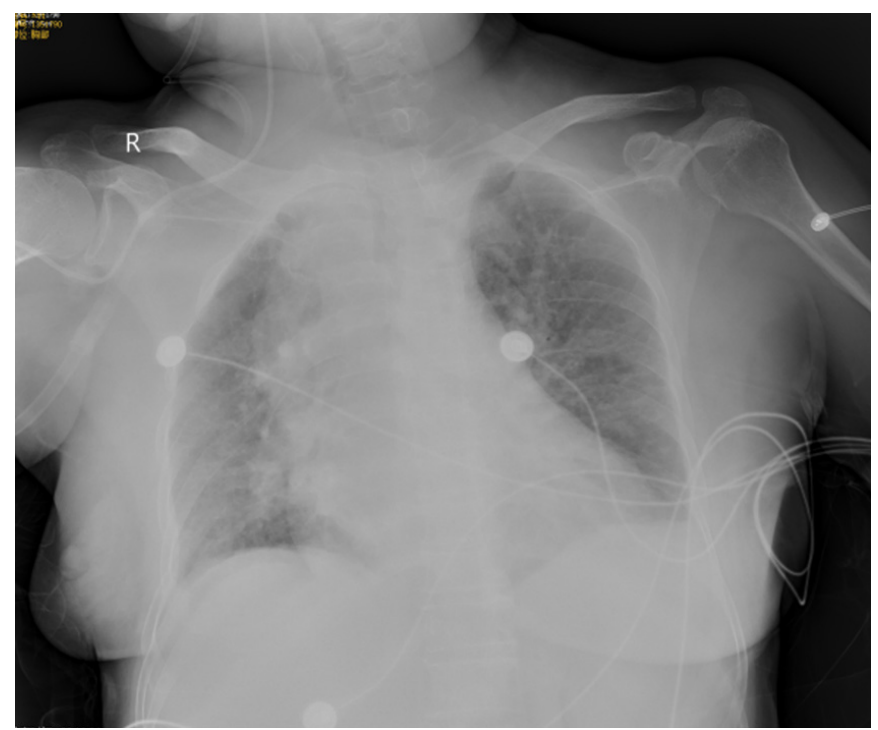

Figure 2. Chest x-ray on day 1 after admission.

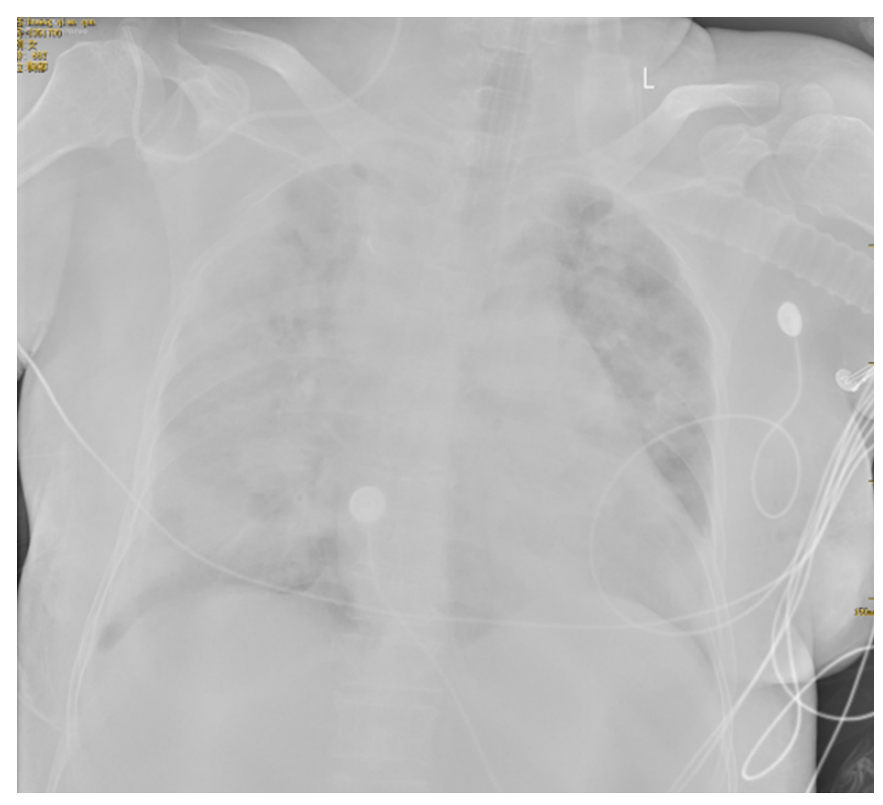

Figure 3. Chest x-ray on day 4 after admission: ARDS.

Laboratory blood tests indicated low platelets and impaired liver and kidney function. She was diagnosed with scrub typhus and acute pneumonia.

After admission, the patient was treated with antiinfective, anti-inflammatory, and liver-protective medication. Vasoactive drugs were used to maintain blood pressure (norepinephrine $0.05 \mu \mathrm{g} / \mathrm{kg} \cdot \mathrm{min}$ ). On day 3 after admission, the patient had shortness of breath and hypoxia and was put on a ventilator. On day 4 after admission, the patient's oxygenation was poor. Originally, she was given lung-protective ventilation, and target tidal volume was $6 \mathrm{~mL} / \mathrm{kg}$ of predicted body weight and plateau pressure was $\leq 30 \mathrm{~cm} \mathrm{H}_{2} \mathrm{O}$, but oxygen partial pressure $\left(\mathrm{PaO}_{2}\right)$ was $<50 \mathrm{mmHg}$. Deep sedation was

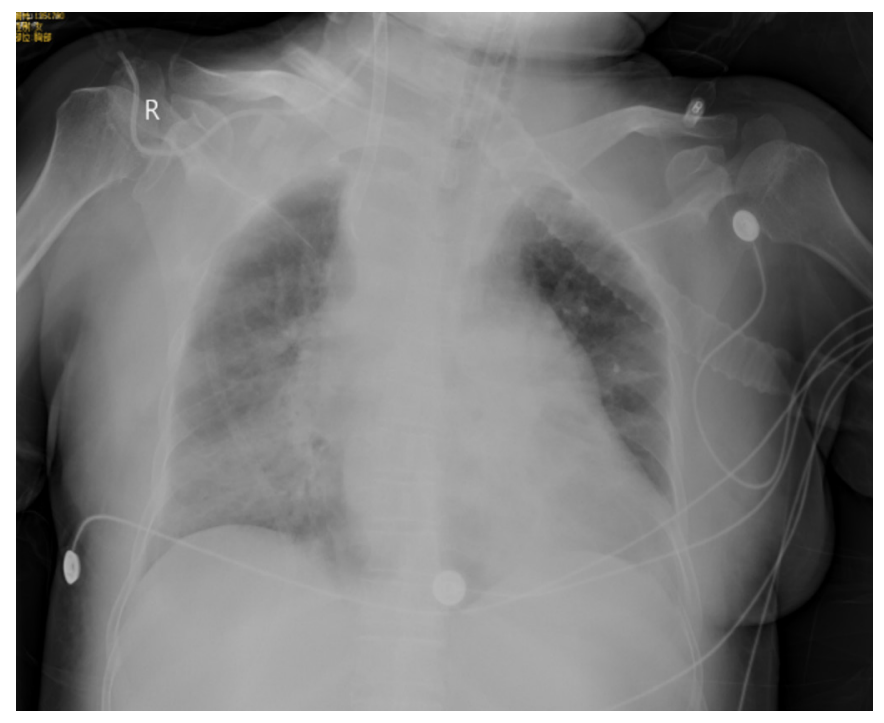

Figure 4. Chest x-ray on day 3 after ECMO assistance.

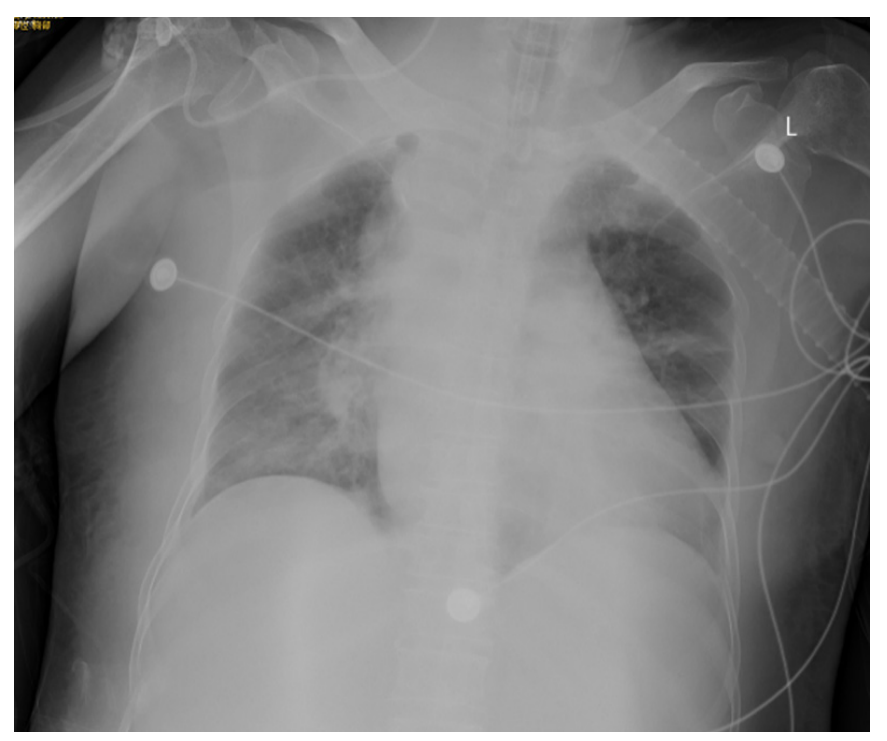

Figure 5. Day 7 of ECMO: chest X-ray after removal of ECMO.

induced with prone positioning, but the ratio of partial pressure to inspired $\mathrm{O}_{2}\left(\mathrm{PaO}_{2} / \mathrm{FiO}_{2}\right)$ was $48 \mathrm{mmHg}$ under high ventilator parameters. High-dose vasoconstrictors (norepinephrine $0.2 \mu \mathrm{g} / \mathrm{kg}$ - min) maintained blood pressure. Echocardiography showed that the heart was functioning normally. The Respiratory ECMO Survival Prediction (RESP) score [Schmidt 2014] was 4 points. Immediately, femoral/internal jugular vein $(\mathrm{V}-\mathrm{V}) \mathrm{ECMO}$ assistance was implemented.

Retrospective data collection was approved by the Institutional Review Board at Zhongshan Hospital affiliated with Sun Yat-Sen University. Written informed consent for publication was obtained, and all data generated or analyzed during this study are included in this published article. 
Blood Gas and Ventilator Parameters Before and After ECMO

\begin{tabular}{|c|c|c|c|c|}
\hline \multicolumn{5}{|l|}{ Blood gas analysis } \\
\hline $\mathrm{CO}_{2}(\mathrm{mmHg})$ & 50.2 & 40 & 35 & 36.9 \\
\hline $\mathrm{PaO}_{2}(\mathrm{mmHg})$ & 48 & 101 & 92 & 100 \\
\hline Base excess & -1 & 1 & -1 & -1.4 \\
\hline \multicolumn{5}{|l|}{ Ventilator parameters } \\
\hline Tidal volume $(\mathrm{mL})$ & 450 & 300 & 450 & 450 \\
\hline Respiratory frequency (bpm) & 14 & 16 & 14 & 14 \\
\hline Positive end expiratory pressure $\left(\mathrm{cm} \cdot \mathrm{H}_{2} \mathrm{O}\right)$ & 15 & 8 & 5 & 5 \\
\hline Flow rate $(\mathrm{L} / \mathrm{min})$ & & 3.5 & 2 & \\
\hline Oxygen concentration (\%) & & 50 & 0 & \\
\hline
\end{tabular}

\section{ECMO}

Device

The ECMO perfusion system consisted of a Biopump centrifugal pump (Medtronic, Dublin, Ireland), Medtronic oxygenator, Stockert SIII temperature-variable water tank (Stockert, Freiburg, Germany), Medtronic Cameda heparincoated ECMO suite, Medtronic intubation, and Medtronic Automated Coagulation Timer (ACT) detector.

\section{System Setup}

The system required $1000 \mathrm{~mL}$ of saline for the precharge exhaust. The patient was systemically heparinized $(1 \mathrm{mg} / \mathrm{kg})$. For V-V ECMO, a 21-Fr (40-cm) Medtronic heparin-coated cannula was inserted into the femoral vein, with a $17-\mathrm{Fr}$ (13$\mathrm{cm}$ ) arterial cannula in the jugular vein.

\section{System Management}

For V-V ECMO, flow of $50-80 \mathrm{~mL} / \mathrm{kg} \cdot \min$ was used for the maintenance of arterial oxygen saturation to levels $>85 \%$ in the lungs at rest. ACT of 160-200 s was maintained through the continuous infusion of heparin.

\section{RESULTS}

Specific blood gas analysis results and ventilator parameters are shown in Figures 1 and 3. On day 3 of ECMO assistance, lung exudation began to be absorbed, as shown in Figure 4. After 7 days of ECMO supplementation, the patient's lung function was significantly improved, blood pressure was stable (no vasoactive drug support), and ECMO was removed (Table 1 and Figure 5). After 3 days, the ventilator was removed. On day 20 after admission, the patient was successfully discharged without any sequelae.

\section{DIsCUSSION}

Scrub typhus is an acute fatal infection caused by tsutsugamushi rickettsia infection in the body. According to 1 study, $\sim 9 \%$ to $25 \%$ of cases with scrub typhus develop into ARDS [Wang 2007]. ARDS is the main predictor of death in patients with scrub typhus [Bhargava 2016]. Even when protective ventilation strategies (ie, tidal volume $6 \mathrm{ml} / \mathrm{kg}$ of predicted body weight and plateau pressure $<30 \mathrm{cmH}_{2} \mathrm{O}$ ) and prone position are used, the mortality rate of ARDS remains high in observational studies [Bellani 2016]. Therefore, to improve prognosis, proper treatment to maintain the body's oxygen supply is very important, which is where ECMO can play a role. In case of ineffective treatment with traditional ventilators, ECMO ensures the oxygen supply of the body. With ECMO support, the ventilator parameters can be reduced, allowing the lungs to rest and recover.

Previous studies have shown that the use of ECMO can improve the survival rate of patients with severe ARDS. Long duration of mechanical ventilation before ECMO and the use of numerous vasopressors may be associated with increased risk of death [Liu 2019] according to RESP scoring recommended by the Extracorporeal Life Support Organization (ELSO). Duration of ventilator treatment $>48$ hours, high peak inspiratory pressure, and incidence of cardiac arrest affect the prognosis of patients with ECMO [Schmidt 2014]. The RESP score of our patient was 4 points, so her predicted 
survival rate was $76 \%$. The timing of ECMO affects the prognosis of patients. Previously, Choi [2016], Oh [2012], and other groups have reported cases of scrub typhus complicated by ARDS that were successfully managed with ECMO.

\section{REFERENCES}

Adhikari S, Paudyal B, Sigdel K R, et al. Meningitis due to scrub typhus: the importance of a differential diagnosis in an endemic area. BMJ Case Rep, 2018,2018.

Bellani G, Laffey J G, Pham T, et al. Epidemiology, Patterns of Care, and Mortality for Patients With Acute Respiratory Distress Syndrome in Intensive Care Units in 50 Countries. JAMA, 2016,315(8):788-800.

Bhargava A, Kaushik R, Kaushik R M, et al. Scrub typhus in Uttarakhand \&amp; adjoining Uttar Pradesh: Seasonality, clinical presentations \&amp; predictors of mortality. Indian J Med Res, 2016,144(6):901-909.

Choi W Y, Lee S Y, Kwon H Y, et al. A Case of Scrub Typhus Complicated by Adult Respiratory Distress Syndrome and Successful Management with Extracorporeal Membrane Oxygenation. Am J Trop Med Hyg, 2016,95(3):554-557.

Fichtner F, Moerer O, Laudi S, et al. Mechanical Ventilation and Extracorporeal Membrane Oxygena tion in Acute Respiratory Insufficiency. Dtsch Arztebl Int, 2018,115(50):840-847.

Jayaprakash V, Vamsikrishna M, Indhumathi E, et al. Scrub typhus-associated acute kidney injury: A study from a South Indian Tertiary Care Hospital. Saudi J Kidney Dis Transpl, 2019,30(4):883-890.

Jeong Y J, Kim S, Wook Y D, et al. Scrub typhus: clinical, pathologic, and imaging findings. Radiographics, 2007,27(1):161-172.

Kelly D J, Fuerst P A, Ching W M, et al. Scrub typhus: the geographic distribution of phenotypic and genotypic variants of Orientia tsutsugamushi. Clin Infect Dis, 2009,48 Suppl 3:S203-S230.

Ki Y J, Kim D M, Yoon N R, et al. A case report of scrub typhus complicated with myocarditis and rhabdomyolysis. BMC Infect Dis, 2018,18(1):551.

Kundavaram AP, Jonathan AJ, Nathaniel S D, et al. Eschar in scrub typhus: a valuable clue to the diagnosis. J Postgrad Med, 2013,59(3):177-178.

Liu S Q, Huang Y Z, Pan C, et al. Venovenous extra-corporeal membrane oxygenation for severe acute respiratory distress syndrome: a matched cohort study. Chin Med J (Engl), 2019,132(18):2192-2198.

Oh W S, Yie K. Extracorporeal membrane oxygenation for extremely complicated scrub typhus. ASAIO J, 2012,58(5):540-541.

Premaratna R, Ariyaratna N, Botheju W I, et al. Scrub typhus pneumonitis. Int J Infect Dis, 2013,17(4):e284.

Ra S H, Kim J Y, Cha H H, et al. Coinfection of Severe Fever with Thrombocytopenia Syndrome and Scrub Typhus in Patients with TickBorne Illness. Am J Trop Med Hyg, 2019.

Schmidt M, Bailey M, Sheldrake J, et al. Predicting survival after extracorporeal membrane oxygenation for severe acute respiratory failure. The Respiratory Extracorporeal Membrane Oxygenation Survival Prediction (RESP) score. Am J Respir Crit Care Med, 2014,189(11):1374-1382.

Song S W, Kim K T, Ku Y M, et al. Clinical role of interstitial pneumonia in patients with scrub typhus: a possible marker of disease severity. J Korean Med Sci, 2004,19(5):668-673.

Vajpayee S, Gupta R K, Gupta M L. Scrub typhus causing neonatal hepatitis with acute liver failure-A case series. Indian J Gastroenterol, 2017,36(3):239-242.

Wang C C, Liu S F, Liu J W, et al. Acute respiratory distress syndrome in scrub typhus. Am J Trop Med Hyg, 2007,76(6):1148-1152. 